\section{Análise da cobertura do Sistema de Informações sobre Mortalidade em Olinda, Pernambuco, Brasil}

\author{
Analysis of coverage in the Mortality Information \\ System in Olinda, Pernambuco State, Brazil
}

\author{
Análisis de cobertura del Sistema de Información \\ sobre Mortalidad en Olinda, Pernambuco, Brasil
}

Barbara de Queiroz Figueiroa 1 Lygia Carmen de Moraes Vanderlei 2 Paulo Germano de Frias 2 Patricia Ismael de Carvalho 1 Celia Landmann Szwarcwald 3

\title{
Resumo
}

\footnotetext{
1 Secretaria de Saúde do Estado de Pernambuco, Recife, Brasil.

2 Instituto de Medicina Integral Prof. Fernando Figueira, Recife, Brasil.

3 Instituto de Comunicação

e Informação Científica

e Tecnológica em Saúde,

Fundação Oswaldo Cruz, Rio

de Janeiro, Brasil.

Correspondência

B. Q. Figueiroa

Secretaria de Saúde do Estado

de Pernambuco.

Rua Dona Maria Augusta

Nogueira 519, Recife, PE

50751-530, Brasil.

barbarafigueiroa@gmail.com
}

\begin{abstract}
This article analyzes the coverage of the Mortality Information System (SIM) in Olinda, Pernambuco State, Brazil, in the year 2008. The study involved secondary data from SIM on deaths (excluding stillbirths) in residents of the municipality and primary data from the Active Search of Deaths and Births in the Northeast and Legal Amazonia, which collected the events from multiple sources and located deaths that had not been reported to the system. Coverage was calculated as the number of deaths recorded in SIM divided by the total (SIM + active search). The study showed $94.8 \%$ coverage and detected an important contribution by notary public offices to the identification of deaths that were missing from the SIM. Of these unreported deaths, $29.7 \%$ occurred in health services, 49\% occurred at home with death certificates signed by private physicians, and $25.5 \%$ had been attested by the forensic examiner's office. The method allowed calculating the coverage rate for the Mortality Information System in a municipality in the metropolitan area. Despite the low proportion of deaths missing in the system, the study detected problems with data collection and flow.
\end{abstract}

Vital Statistics; Information Systems; Mortality
Neste trabalho, analisa-se a cobertura do Sistema de Informações sobre Mortalidade (SIM) em Olinda, Pernambuco, Brasil, no ano de 2008. O estudo envolveu dados secundários sobre óbitos não fetais de residentes do município obtidos do SIM e dados primários da pesquisa Busca Ativa de Óbitos e Nascimentos no Nordeste e Amazônia Legal, que coletou os eventos em múltiplas fontes e localizou óbitos não informados ao sistema. A cobertura foi representada pela proporção de óbitos constantes no SIM em relação ao total informado (SIM + busca ativa). O estudo identificou 94,8\% de cobertura e observou a importante contribuição dos cartórios para o conhecimento dos óbitos ausentes no SIM. Desses, 29,7\% ocorreram em estabelecimentos de saúde; $49 \%$ ocorreram em domicílio e foram atestados por médicos particulares; $e$ 25,5\% do total de óbitos localizados foram atestados pelo IML. O método aplicado permitiu identificar a cobertura do SIM em município de região metropolitana. Apesar da pequena proporção de óbitos ausentes no SIM, o estudo sinalizou problemas relacionados à coleta e fluxo.

Estatísticas Vitais; Sistemas de Informação; Mortalidade 


\section{Introdução}

Os sistemas de informações de registro contínuo são úteis para elaboração de perfis epidemiológicos, para o planejamento de ações e para a organização de serviços de saúde de acordo com as necessidades da população 1 .

No Brasil, com a implantação do Sistema de Informações sobre Mortalidade (SIM), em 1975, ocorreu o avanço das estatísticas de mortalidade pela padronização de registros dos dados de óbito por meio da Declaração de Óbito (DO) 2 . Entretanto, a existência de óbitos não informados ao SIM, em especial em alguns municípios das regiões Norte e Nordeste, restringe o uso do sistema, pois dificulta a obtenção de dados diretos com regularidade, qualidade e cobertura adequada $^{3}$.

Atentos à importância dos sistemas de informações para garantir dados confiáveis, vêm sendo realizados estudos sobre sua qualidade, a exemplo da avaliação do SIM em múltiplas perspectivas, incluindo a cobertura do sistema 4 .

A cobertura pode ser analisada por meio de diversas técnicas de estimação, tais como: relacionamento entre registros 5 ; consistência de indicadores do próprio banco de dados 6; razão entre o número de eventos captados e estimados, mediante técnicas que utilizam dados do próprio sistema ou de outros 7 , e a busca ativa de eventos 8,9

O Ministério da Saúde incentiva o uso de técnicas de estimação do número de óbitos para avaliar a cobertura do SIM, porém existem dificuldades metodológicas, particularmente para pequenas áreas como vários municípios do Brasil 7,10. Dessa forma, alguns estudos privilegiaram a metodologia da busca ativa de óbitos em múltiplas fontes, limitando-se, no entanto, aos municípios de pequeno porte populacional com informações insatisfatórias 9,11.

Com a realização da Pesquisa de Busca Ativa de Óbitos e Nascimentos no Nordeste e Amazônia Legal, doravante chamada Busca Ativa, desenvolvida pela Fundação Oswaldo Cruz (Fiocruz) e Ministério da Saúde 12, e a disponibilidade de informações sobre óbitos em municípios sorteados por amostra probabilística, foi possível estimar a cobertura de óbitos em municípios de grande porte populacional.

Com base nessa pesquisa, este trabalho objetivou avaliar a cobertura do SIM do Município de Olinda, Pernambuco, um dos cinco sorteados aleatoriamente para compor o estrato Nordeste, com informações vitais adequadas e com população maior que 200 mil habitantes, e descrever as características sociodemográficas dos óbitos não informados ao sistema.

\section{Metodologia}

O Município de Olinda, contíguo à capital do estado, tem uma área de $40,83 \mathrm{~km}^{2}$ e população de 397.268 habitantes, com taxa de urbanização de 98\%, sendo a densidade demográfica de 13.497 habitantes $/ \mathrm{km}^{2}$. Composta por 32 bairros e dois Distritos Sanitários, é a terceira cidade mais populosa de Pernambuco (Instituto Brasileiro de Geografia e Estatística. Censo demográfico 2000. http://www.ibge.gov.br).

A população do estudo envolveu o universo dos óbitos não fetais de residentes do município ocorridos no ano de 2008. Foram utilizados dados secundários de óbitos da base de dados do SIM municipal e estadual e dados primários da pesquisa de Busca Ativa ${ }^{12}$, cuja coleta ocorreu entre os meses de setembro de 2009 e junho de 2010.

A pesquisa de Busca Ativa identificou os óbitos fetais e não fetais de residentes dos municípios selecionados em amostra probabilística, ocorridos em 2008 não informados ao SIM, e coletados em diversas fontes de informação por profissionais de saúde previamente capacitados. A inclusão dos óbitos fetais na busca ativa objetivou identificar erros na classificação quanto ao tipo de óbito relacionando como fetais os não fetais.

Entretanto, para o cálculo da cobertura do SIM, foram utilizados apenas os óbitos não fetais, excluindo-se os óbitos fetais pela dificuldade de precisar a idade gestacional limítrofe entre aborto e óbito fetal.

Em Olinda, a busca ativa foi realizada nas seguintes fontes localizadas no próprio município e na capital, Recife: cartórios de registro civil e estabelecimentos de saúde; cemitérios, Serviços de Transporte de Pacientes (STP), funerárias, unidades básicas de saúde (UBS), abrigos/asilos; Instituto Médico Legal (IML) e Serviço de Verificação de Óbito (SVO).

Os óbitos identificados nas diversas fontes e que não constavam na base de dados do SIM, foram confirmados pelo resgate das cópias das DO, das certidões de óbito dos cartórios de registro civil ou das cópias da DO dos estabelecimentos de saúde, ou por intermédio das entrevistas domiciliares quando não se localizaram tais documentos.

Foi elaborado um banco de dados em meio eletrônico dos óbitos não fetais com as seguintes informações: nome do falecido, data do óbito, número da DO, nome da mãe, endereço de residência, bairro, município, confirmação do caso, fonte de coleta do óbito e fonte de confirmação.

A cobertura foi representada pela proporção de óbitos localizados no sistema em relação ao 
total (óbitos não fetais de residentes de Olinda localizados no SIM acrescidos dos encontrados na busca ativa). Os óbitos ausentes no SIM que foram localizados na busca ativa corresponderam ao sub-registro. Para eles foi descrito o perfil sociodemográfico contendo sexo, faixa etária, raça/ cor, escolaridade, ocupação, estado civil e causa básica de óbito segundo capítulo da 10a revisão da Classificação Internacional das Doenças (CID10). Utilizou-se o teste exato de Fisher pelo programa Stata (Stata Corp., College Station, Estados Unidos) para comparar as proporções das variáveis com exclusão das informações ignoradas.

Para identificar a contribuição de cada fonte de informação, considerou-se a razão entre os óbitos localizados em cada uma delas e o total de óbitos não informados ao SIM. A concomitância na localização dos óbitos nas múltiplas fontes foi observada com base no número absoluto de óbitos existentes em cada fonte, assim como se identificaram os óbitos que eram exclusivos de uma única fonte.

Para caracterizar a situação de cada óbito informado ou não ao SIM, foram considerados: (a) locais de ocorrência do óbito - estabelecimentos de saúde, domicílio, via pública, outros e ignorado; (b) instituições emissoras da DO - estabelecimentos de saúde, IML, SVO e médicos particulares para os óbitos domiciliares. Quando não foi possível identificar a fonte emissora, utilizou-se a classificação “desconhecido”.
Igualmente, para discriminar a fonte emissora da DO segundo o local de ocorrência dos óbitos não informados ao SIM, apresentou-se um fluxograma caracterizando o número e proporção de DO emitida por cada fonte, considerando o total de óbitos ocorridos. Para essa situação, foi possível incorporar a informação quanto aos asilos e abrigos não disponível no SIM (Figura 1).

O projeto foi submetido e aprovado pelo Comitê de Ética em Pesquisa do Instituto de Medicina Integral Prof. Fernando Figueira (IMIP).

\section{Resultados}

O total de óbitos não fetais de residentes do $\mathrm{Mu}$ nicípio de Olinda em 2008 foi 2.795. O SIM apresentou uma cobertura de $94,8 \%$, o que correspondeu a 2.650 óbitos não fetais. O número de óbitos não informados ao sistema e localizados por busca ativa foi de 145 (5,2\%).

Os cartórios de registro civil representaram a principal contribuição para melhoria da cobertura do SIM, sendo possível identificar 93,8\% do total de óbitos não informados ao sistema, seguidos pelos cemitérios, IML e estabelecimentos de saúde (Tabela 1).

Observou-se a preponderância dos cartórios na concomitância da localização dos óbitos nas múltiplas fontes. Dos 136 óbitos localizados neles, foram encontrados também nas seguintes
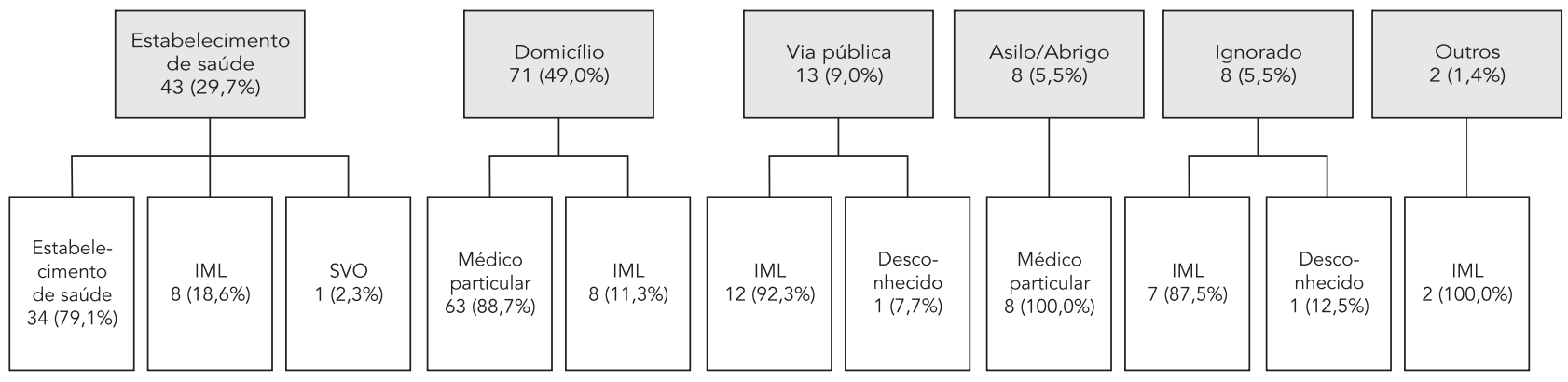

Local de ocorrência

Fontes emissoras da DO

IML: Instituto Médico Legal; SVO: serviço de verificação de óbito. 
Distribuição dos óbitos não informados ao Sistema de Informações sobre Mortalidade (SIM), segundo fontes de informação. Olinda, Pernambuco, Brasil, 2008.

\begin{tabular}{|c|c|c|}
\hline \multirow[t]{2}{*}{ Fontes de informação } & \multicolumn{2}{|c|}{ Óbitos não fetais ( $N$ = 145) } \\
\hline & $n$ & $\%$ \\
\hline Cartórios & 136 & 93,8 \\
\hline Cemitérios & 41 & 28,3 \\
\hline IML & 33 & 22,8 \\
\hline Estabelecimentos de saúde & 30 & 20,7 \\
\hline Serviço de transporte de pacientes & 10 & 6,9 \\
\hline Funerárias & 6 & 4,1 \\
\hline Abrigos e asilos & 5 & 3,4 \\
\hline UBS & 4 & 2,8 \\
\hline SVO & 1 & 0,7 \\
\hline
\end{tabular}

IML: Instituto Médico Legal; SVO: serviço de verificação de óbito; UBS: unidades básicas de saúde.

Nota: a soma não totaliza 100\% porque um mesmo óbito pode ter sido encontrado em mais de uma fonte pesquisada.

fontes: 40 nos cemitérios; 27 no IML; 27 nos estabelecimentos de saúde; nove nos STP; seis nas funerárias; cinco nos abrigos/asilos e um na UBS e no SVO. Em uma única fonte foram encontrados 50 óbitos nos cartórios de registro civil, cinco no IML, dois nos estabelecimentos de saúde e um nos STP (Tabela 2).

Em relação ao local de ocorrência, o maior número de óbitos não informados ao SIM foi domiciliar (49\%), com um aumento de $12,7 \%$ para 14,6\% quando acrescidos aos óbitos informados ao SIM. Quanto à fonte emissora, encontrou-se que os médicos particulares foram responsáveis por emitir 49\% das DO dos óbitos não informados, seguidos do IML e estabelecimentos de saúde $(25,5 \%$ e $23,4 \%$, respectivamente) (Tabela 3).

Dos 43 óbitos ocorridos nos estabelecimentos de saúde, $34(79,1 \%)$ tiveram a DO emitida pelo próprio estabelecimento, 8 (18,6\%) no IML e 1 (2,3\%) pelo SVO. Dos 71 óbitos ocorridos no domicílio, $63(88,7 \%)$ foram atestados por médico particular e $8(11,3 \%)$ por profissionais do IML. Permaneceram desconhecidas as fontes emissoras de duas DO, cujos óbitos ocorreram em via pública e em local ignorado (Figura 1).

Quanto às variáveis socioeconômicas, o perfil dos óbitos não informados mostra que 50,3\% eram do sexo feminino, $65,5 \%$ encontravam-se na faixa etária $\geq 60$ anos, $91 \%$ eram distribuídos igualmente entre negras e brancas; $15 \%$ dos óbitos tinham de 4 a 7 anos de estudo concluídos, mais de 30,3\% eram aposentados/pensionistas e $36,6 \%$ eram solteiros. Enquanto para os óbitos informados predominou o sexo masculino
(54,9\%), mesma faixa etária dos não informados com maior percentual de negros $(61,2 \%)$. Quanto à escolaridade e ocupação, verificou-se um elevado percentual de ignorados, mais acentuado entre os óbitos informados ao sistema. Em relação às causas básicas de óbito com o acréscimo da busca ativa não se verificaram mudanças expressivas na mortalidade proporcional por causa, capítulo da CID-10. Apenas as variáveis raça/cor e causas básicas de óbito foram estatisticamente significantes (Tabela 4).

Em 2008, o coeficiente geral de mortalidade (CGM) de Olinda era de 6,7 óbitos por mil habitantes, elevando-se para 7,1 ao se agregarem os óbitos identificados na busca ativa, enquanto o coeficiente de mortalidade infantil (CMI) cresceu de 15,8 por mil nascidos vivos para 16,1 .

\section{Discussão}

O estudo mostrou que o método utilizado suplantou as limitações de conhecimento da cobertura para desagregados geográficos maiores e permitiu identificar que o Município de Olinda apresenta uma ampla cobertura do SIM, que possibilita utilizar os dados dispostos no sistema ${ }^{1}$.

Tal resultado reveste-se de importância ao considerar o acentuado interesse da gestão em melhorar a qualidade dos dados no nível local $13,14,15$, acrescido da pouca disponibilidade de metodologia adequada para identificar a cobertura do SIM no âmbito municipal, sobretudo por meio de técnicas que a expressem fidedignamente 9,16 . 
Número de óbitos não informados ao Sistema de Informações sobre Mortalidade (SIM), segundo múltiplas fontes de informação. Olinda, Pernambuco, Brasil, 2008.

\begin{tabular}{|c|c|c|c|c|c|c|c|c|c|}
\hline Fontes de informação & $\begin{array}{l}\text { Cartório } \\
(n=50) \text { * }\end{array}$ & $\begin{array}{c}\text { SVO } \\
(n=0) \text { * }\end{array}$ & $\begin{array}{c}\text { IML } \\
(n=5) \text { * }\end{array}$ & $\begin{array}{l}\text { Estabeleci- } \\
\text { mentos de } \\
\text { saúde } \\
(n=2) \text { * }\end{array}$ & $\begin{array}{c}\text { STP } \\
(n=1) \text { * }\end{array}$ & $\begin{array}{c}\text { UBS } \\
(n=0) \text { * }\end{array}$ & $\begin{array}{l}\text { Cemitérios } \\
\qquad(\mathrm{n}=0) \text { * }\end{array}$ & $\begin{array}{l}\text { Funerárias } \\
(n=0) \text { * }\end{array}$ & $\begin{array}{c}\text { Abrigos e } \\
\text { asilos } \\
(n=0) \text { * }\end{array}$ \\
\hline Cartório & 136 & 1 & 27 & 27 & 9 & 1 & 40 & 6 & 5 \\
\hline SVO & 1 & 1 & 0 & 1 & 0 & 0 & 1 & 0 & 0 \\
\hline IML & 27 & 0 & 33 & 3 & 2 & 4 & 10 & 0 & 0 \\
\hline Estabelecimentos de saúde & 27 & 1 & 3 & 30 & 1 & 1 & 14 & 1 & 0 \\
\hline STP & 9 & 0 & 2 & 1 & 10 & 0 & 3 & 1 & 0 \\
\hline UBS & 1 & 0 & 4 & 1 & 0 & 4 & 1 & 0 & 0 \\
\hline Cemitérios & 40 & 1 & 10 & 14 & 3 & 1 & 41 & 0 & 0 \\
\hline Funerárias & 6 & 0 & 0 & 1 & 1 & 0 & 0 & 6 & 0 \\
\hline Abrigos e asilos & 5 & 0 & 0 & 0 & 0 & 0 & 0 & 0 & 5 \\
\hline
\end{tabular}

IML: Instituto Médico Legal; STP: serviço de transporte de paciente; SVO: serviço de verificação de óbito; UBS: unidades básicas de saúde.

* Os valores entre parênteses representam o número de óbitos localizados exclusivamente em cada fonte de informação.

Nota: os dados da linha e coluna não correspondem ao total de óbitos identificados na busca ativa porque o mesmo óbito pode ser encontrado em mais de uma fonte.

Tabela 3

Distribuição dos óbitos não informados e informados ao Sistema de Informações sobre Mortalidade (SIM), segundo local de ocorrência e fonte emissora da Declaração de Óbito (DO). Olinda, Pernambuco, Brasil, 2008.

\begin{tabular}{|c|c|c|c|c|c|c|}
\hline \multirow[t]{2}{*}{ Variáveis } & \multicolumn{2}{|c|}{ Não informados ao SIM } & \multicolumn{2}{|c|}{ Informados ao SIM } & \multicolumn{2}{|c|}{ Total } \\
\hline & $\mathrm{n}$ & $\%$ & $\mathbf{n}$ & $\%$ & $\mathbf{n}$ & $\%$ \\
\hline \multicolumn{7}{|l|}{ Local de ocorrência } \\
\hline Estabelecimento de saúde & 43 & 29,7 & 2.073 & 78,2 & 2.116 & 75,7 \\
\hline Domicílio & 71 & 49,0 & 337 & 12,7 & 408 & 14,6 \\
\hline Via pública & 13 & 9,0 & 206 & 7,8 & 219 & 7,8 \\
\hline Outros * & 10 & 6,9 & 27 & 1,0 & 37 & 1,0 \\
\hline Ignorado & 8 & 5,5 & 7 & 0,3 & 15 & 0,5 \\
\hline \multicolumn{7}{|l|}{ Fonte emissora da DO } \\
\hline Estabelecimento de saúde & 34 & 23,4 & 1.418 & 53,5 & 1.452 & 51,9 \\
\hline Médico particular & 71 & 49,0 & 66 & 2,5 & 137 & 4,9 \\
\hline IML & 37 & 25,5 & 435 & 16,4 & 472 & 16,9 \\
\hline SVO & 1 & 0,7 & 717 & 27,1 & 718 & 25,7 \\
\hline Desconhecido/ignorado & 2 & 1,4 & 14 & 0,5 & 16 & 0,6 \\
\hline
\end{tabular}

IML: Instituto Médico Legal; SVO: serviço de verificação de óbito.

* Incorporados os dados referentes aos abrigos/asilos.

Para tanto, o Ministério da Saúde vem instruindo os estados a observarem a cobertura do sistema nos municípios pelo CGM, e pactuam ações para melhoria de captação de óbitos 17 sob o parâmetro fundamentado no estudo de Andrade \& Szwarcwald 3.
Contudo, os critérios estabelecidos foram desenvolvidos para o Brasil, sendo mais úteis para identificar municípios com grande precariedade de informação e, ao mesmo tempo, apresentam limitações para identificar o grau da adequação dos municípios com informações satisfatórias 3,18. 
Distribuição dos óbitos não informados e informados ao Sistema de Informações sobre Mortalidade (SIM), segundo variáveis sociodemográficas e causa básica de óbito de acordo com a 10ạ revisão da Classificação Internacional de Doenças (CID-10). Olinda, Pernambuco, Brasil, 2008.

\begin{tabular}{|c|c|c|c|c|c|c|c|}
\hline \multirow[t]{2}{*}{ Variáveis } & \multicolumn{2}{|c|}{$\begin{array}{l}\text { Não informados } \\
\text { ao SIM }\end{array}$} & \multicolumn{2}{|c|}{$\begin{array}{l}\text { Informados ao } \\
\text { SIM }\end{array}$} & \multirow[t]{2}{*}{$\begin{array}{l}\text { Valor } \\
\text { de } p \text { * }\end{array}$} & \multicolumn{2}{|c|}{ Total } \\
\hline & $\mathrm{n}$ & $\%$ & $n$ & $\%$ & & $\mathrm{n}$ & $\%$ \\
\hline \multicolumn{8}{|l|}{ Sexo } \\
\hline Masculino & 72 & 49,7 & 1.455 & 54,9 & - & 1.527 & 54,6 \\
\hline Feminino & 73 & 50,3 & 1.194 & 45,1 & 0,231 & 1.267 & 45,4 \\
\hline Ignorado & 0 & - & 1 & - & - & 1 & - \\
\hline \multicolumn{8}{|c|}{ Faixa etária/Ciclos de vida (anos) } \\
\hline$<1$ & 2 & 1,4 & 95 & 3,6 & - & 97 & 3,5 \\
\hline $1-9$ & 0 & 0,0 & 19 & 0,7 & - & 19 & 0,7 \\
\hline $10-19$ & 6 & 4,2 & 88 & 3,3 & - & 94 & 3,4 \\
\hline $20-39$ & 19 & 13,2 & 333 & 12,6 & - & 352 & 12,6 \\
\hline $40-59$ & 22 & 15,3 & 575 & 21,7 & - & 597 & 21,4 \\
\hline $60 e+$ & 95 & 66,0 & 1.536 & 58,0 & 0,221 & 1.631 & 58,4 \\
\hline Ignorado & 1 & - & 4 & - & - & 5 & - \\
\hline \multicolumn{8}{|l|}{ Raça/Cor } \\
\hline Branca & 66 & 50,0 & 822 & 33,5 & - & 888 & 31,8 \\
\hline Negra & 66 & 50,0 & 1.621 & 66,1 & - & 1687 & 60,4 \\
\hline Demais & 0 & 0,0 & 8 & 0,3 & 0,001 & 8 & 0,3 \\
\hline Ignorado & 13 & - & 199 & - & - & 212 & - \\
\hline \multicolumn{8}{|c|}{ Escolaridade (anos concluídos) ** } \\
\hline Nenhuma & 14 & 16,9 & 142 & 13,5 & - & 156 & 5,6 \\
\hline $1-3$ & 18 & 21,7 & 192 & 18,2 & - & 210 & 7,5 \\
\hline $4-7$ & 23 & 27,6 & 384 & 36,3 & - & 407 & 14,6 \\
\hline 8-11 & 16 & 19,3 & 194 & 18,4 & - & 210 & 7,5 \\
\hline $12 \mathrm{e}+$ & 12 & 14,5 & 143 & 13,6 & - & 155 & 5,5 \\
\hline Ignorado & 62 & - & 1.595 & - & - & 1.657 & - \\
\hline \multicolumn{8}{|l|}{ Ocupação ** } \\
\hline Aposentado/Pensionista & 44 & 56,5 & 3 & 33,4 & - & 47 & 1,7 \\
\hline Dona de casa & 22 & 28,2 & 4 & 44,4 & - & 26 & 0,9 \\
\hline Estudante & 3 & 3,8 & 1 & 11,1 & - & 4 & 0,1 \\
\hline Demais & 9 & 11,5 & 1 & 11,1 & - & 10 & 0,4 \\
\hline Ignorado & 67 & - & 2.641 & - & - & 2.708 & - \\
\hline \multicolumn{8}{|l|}{ Estado civil } \\
\hline Solteiro & 53 & 42,8 & 969 & 41,6 & - & 1.022 & 36,6 \\
\hline Casado & 39 & 31,4 & 816 & 35,0 & - & 855 & 30,6 \\
\hline Viúvo & 29 & 23,4 & 480 & 20,6 & - & 509 & 18,2 \\
\hline União estável & 3 & 2,4 & 66 & 2,8 & 0,813 & 69 & 2,5 \\
\hline Ignorado & 21 & - & 319 & - & - & 340 & - \\
\hline
\end{tabular}

(continua) 


\begin{tabular}{|c|c|c|c|c|c|c|c|}
\hline \multirow[t]{2}{*}{ Variáveis } & \multicolumn{2}{|c|}{$\begin{array}{l}\text { Não informados } \\
\text { ao SIM }\end{array}$} & \multicolumn{2}{|c|}{$\begin{array}{l}\text { Informados ao } \\
\text { SIM }\end{array}$} & \multirow[t]{2}{*}{$\begin{array}{l}\text { Valor } \\
\text { de } p \text { * }\end{array}$} & \multicolumn{2}{|c|}{ Total } \\
\hline & n & $\%$ & n & $\%$ & & $\mathbf{n}$ & $\%$ \\
\hline \multicolumn{8}{|l|}{ Causas (capítulo da CID-10) } \\
\hline \multicolumn{8}{|l|}{ Causas definidas } \\
\hline Doenças infecciosas & 7 & 4,8 & 135 & 5,1 & - & 142 & 5,1 \\
\hline Neoplasias & 9 & 6,2 & 385 & 14,5 & - & 394 & 14,1 \\
\hline Doenças do sangue e dos & 0 & 0,0 & 11 & 0,4 & - & 11 & 0,4 \\
\hline $\begin{array}{l}\text { órgãos hematopoéticos e alguns } \\
\text { transtornos imunitários }\end{array}$ & & & & & & & \\
\hline $\begin{array}{l}\text { Doenças endócrinas nutricionais e } \\
\text { metabólicas }\end{array}$ & 21 & 14,5 & 143 & 5,4 & - & 164 & 5,9 \\
\hline $\begin{array}{l}\text { Transtornos mentais e } \\
\text { comportamentais }\end{array}$ & 1 & 0,7 & 22 & 0,8 & - & 23 & 0,8 \\
\hline Doenças do sistema nervoso & 5 & 3,4 & 35 & 1,3 & - & 40 & 1,4 \\
\hline Doenças do aparelho circulatório & 37 & 25,5 & 850 & 32,1 & - & 887 & 31,7 \\
\hline Doenças do aparelho respiratório & 18 & 12,4 & 298 & 11,2 & - & 316 & 11,3 \\
\hline Doenças do aparelho digestivo & 1 & 0,7 & 171 & 6,5 & - & 172 & 6,2 \\
\hline $\begin{array}{l}\text { Doenças da pele e do tecido } \\
\text { subcutâneo }\end{array}$ & 0 & 0,0 & 4 & 0,2 & - & 4 & 0,1 \\
\hline Doenças sistema osteomuscular & 0 & 0,0 & 6 & 0,2 & - & 6 & 0,2 \\
\hline $\begin{array}{l}\text { Doenças do aparelho } \\
\text { geniturinário }\end{array}$ & 1 & 0,7 & 89 & 3,4 & - & 90 & 3,2 \\
\hline $\begin{array}{l}\text { Doenças relacionadas à gravidez, } \\
\text { parto e puerpério }\end{array}$ & 0 & 0,0 & 5 & 0,2 & - & 5 & 0,2 \\
\hline Afecções do período perinatal & 1 & 0,7 & 56 & 2,1 & - & 57 & 2,0 \\
\hline Malformação congênita & 0 & 0,0 & 24 & 0,9 & - & 24 & 0,9 \\
\hline Causas externas & 28 & 19,3 & 392 & 14,8 & - & 420 & 15,0 \\
\hline Causas mal definidas & 16 & 11,0 & 24 & 0,9 & 0,001 & 40 & 1,4 \\
\hline
\end{tabular}

* Teste exato de Fisher excluindo os ignorados;

** Valor de p não calculado.

Nesse cenário, os gestores seguem enfrentando obstáculos para conhecer, monitorar e avaliar a cobertura do SIM local, e a busca ativa de óbitos parece ser uma boa alternativa, considerando que outros estudos limitaram-se a identificar a cobertura para outros âmbitos geográficos, ou mesmo para faixa etária específica $9,10,11,16,19$.

De acordo com o esperado, e por ser uma fonte tradicional de referência em aglomerados urbanos também priorizados para estudos realizados no âmbito estadual, os cartórios contribuíram com quase a totalidade do resgate da busca ativa 19. É importante que se tenha em conta o número expressivo de óbitos domiciliares não informados ao SIM, pois enaltecem a importância dos cartórios no resgate de óbitos ausentes no sistema e suas respectivas DO, e evidenciam problemas relacionados à coleta, processamento e fluxo.
Em contraponto, estudos realizados em municípios de menor porte populacional, ou em agregados deles, constataram expressivo número de óbitos sem emissão de DO ou sinalizaram para a incerteza da emissão do instrumento 10,11 , caracterizando-se com a disposição de áreas com deficiência de cobertura dos serviços de saúde, zonas rurais e presença de cemitérios irregulares, que refletem maior número de sub-registro dos eventos vitais 20,21 .

Estudo realizado em região metropolitana no Ceará 21 observou o elevado número de óbitos identificados em cemitérios e não informados ao SIM, diferentemente dos achados em Olinda que não se mostraram importantes fontes de busca, pois quase a totalidade dos óbitos encontrados nesta fonte, foi localizada nos cartórios de registro civil.

A não identificação dos óbitos em cemitérios como fonte exclusiva de eventos não informados 
ao SIM pode estar revelando o cumprimento dos trâmites legais relacionados ao sepultamento, pois não existem cemitérios irregulares no município. Todavia, foi evidenciado o desconhecimento do fluxo das vias da DO, bem como da relevância da informação para fins epidemiológicos por parte dos profissionais médicos ou mesmo das instituições oficiais emissoras da DO 22.

Ainda que a proporção de óbitos ausentes no SIM resgatados nos estabelecimentos de saúde seja pequena, reveste-se de grande importância por evidenciar o nível de organização e a qualidade dos serviços 23 e por pressupor a falta de integração entre os estabelecimentos de saúde e a gestão do SIM municipal, demonstrando dificuldades na coleta do instrumento nessa fonte de informação oficial 24 .

Igualmente, a contribuição do IML como fonte de informação na busca ativa desempenhou destacada participação na identificação de óbitos não informados ao SIM, refletindo a fragilidade municipal no estabelecimento do fluxo da DO emitida por este serviço, situado na capital do estado, achados similares a outro estudo 6 .

Uma vez que legalmente o sistema preconiza que os óbitos devem ser coletados e digitados em tempo oportuno pelo município de ocorrência 25,26, é necessário maior rigor nos mecanismos que facilitem o envio da 1a via da DO emitida pelo IML para esses municípios, evitando extravios e/ou lentidão no processo.

O produto da busca ativa de óbitos não exerceu mudanças expressivas no perfil sociodemográfico, apesar da concentração dos óbitos não informados ao SIM em grupos populacionais específicos e determinados agravos, como os idosos, as doenças do aparelho circulatório e as causas externas, e do achado de significância estatística para as variáveis raça/cor e causa básica. Parte expressiva desses resultados é semelhante a outros estudos 8,11. Assim como os óbitos informados ao SIM, os não informados apresentam proporção de informação ignorada elevada quanto à escolaridade e ocupação, o que inviabilizou a comparação das proporções e dificultou analisar adequadamente o perfil sociodemográfico, denunciando a necessidade de melhorar a qualidade de preenchimento da DO 27.

Embora exista pequena elevação do CMI e do CGM em Olinda com a incorporação dos óbitos não informados ao SIM, os resultados apro- ximam-se muito do CMI estimado pelo fator de correção para os agregados de municípios da região Nordeste com população de mais de $200 \mathrm{mil}$ habitantes e informações adequadas da pesquisa Busca Ativa 12. Da mesma forma, a cobertura verificada neste estudo foi próxima à estimada pela referida pesquisa 12 .

Os resultados da busca ativa em Olinda conferiram legitimidade aos critérios estabelecidos para classificar a adequação das informações vitais em município de grande porte com informações satisfatórias, aspecto até então avaliado como uma das imprecisões da classificação sugerida $7,15,18$.

Por mais que a metodologia aplicada permita analisar a cobertura do SIM num município de grande porte populacional com informações satisfatórias 18 , algumas limitações relacionadas à dificuldade de operacionalização devem ser consideradas por se tratar de uma região metropolitana que apresenta contíguos territoriais e constante deslocamento intermunicipal. Outras limitações envolvem a não realização da busca ativa em fontes alternativas não selecionadas e a não utilização de outros bancos de dados para relacionar com o SIM.

Saliente-se que as múltiplas fontes poderiam ser utilizadas para o cálculo da estimativa de óbitos empregando outras técnicas como a exemplo da captura-recaptura baseadas na aplicação de modelos 28 , porém não foi possível calcular a cobertura por fonte, uma vez que a busca ativa foi direcionada aos casos não notificados ao SIM e não ao universo de óbitos registrados em cada fonte, sendo uma alternativa viável para futuros trabalhos desde que considere os pressupostos da técnica 29.

O estudo mostrou que o sub-registro ao SIM do Município de Olinda é baixo e que a principal fonte de busca ativa são os cartórios de registro civil. Além disso, possibilitou identificar fragilidades na operacionalização do SIM e sinalizou para possíveis mudanças no processo de trabalho no âmbito local, a exemplo do fortalecimento de parcerias com as fontes de informação, em especial, os próprios cartórios de registro civil. O conhecimento do seu banco de dados e a possibilidade de relacioná-lo com o SIM são atividades que podem viabilizar a aplicação da busca ativa na rotina dos serviços, tornando-a factível e menos onerosa. 


\section{Resumen}

En este trabajo se analiza la cobertura del Sistema de Información sobre Mortalidad (SIM) en Olinda, Pernambuco, Brasil, durante el año 2008. El estudio incluyó datos secundarios sobre óbitos no fetales de residentes del municipio, obtenidos del SIM y datos primarios de la investigación Búsqueda activa de óbitos y nacimientos en el Nordeste y Amazonia legal, que recogió los casos procedentes de múltiples fuentes y localizó óbitos no informados al sistema. La cobertura fue representada por la proporción de óbitos constantes en el SIM, en relación con el total informado (SIM + búsqueda activa). El estudio identificó un $94,8 \%$ de cobertura y observó la importante contribución de los registros civiles para el conocimiento de los óbitos ausentes en el SIM. De ellos, un 29,7\% se produjeron en establecimientos de salud; un 49,0\% se produjeron en domicilios y fueron certificados por médicos privados; y un 25,5\% del total de óbitos localizados fueron certificados por el IML (Instituto Médico Legal). El método aplicado permitió identificar la cobertura del SIM en el municipio de la región metropolitana. A pesar de la pequeña proporción de óbitos ausentes en el SIM, el estudio señaló problemas relacionados con la recogida y flujo de los mismos.

Estadísticas Vitales; Sistemas de Información; Mortalidad

\section{Colaboradores}

B. Q. Figueiroa contribuiu na concepção, pesquisa bibliográfica, construção do banco, análise dos dados e redação do artigo. L. C. M. Vanderlei e P. G. Frias colaboraram na concepção, análise dos dados e redação do artigo. P. I. Carvalho e C. L. Szwarcwald contribuíram na concepção e revisão do artigo.

\section{Agradecimentos}

Ao Ministério da Saúde, Fundação Oswaldo Cruz e Secretaria de Saúde do Estado de Pernambuco pela disponibilização dos dados que possibilitaram a análise. Ao Mestrado Profissional em Avaliação em Saúde do Instituto de Medicina Integral Prof. Fernando Figueira (IMIP) pelo incentivo ao conhecimento científico aplicado à realidade dos serviços de saúde.

\section{Referências}

1. Rede Interagencial de Informações para a Saúde. Indicadores básicos para saúde no Brasil: conceitos e aplicações 2a Ed. Brasília: Organização PanAmericana da Saúde; 2008.

2. Mello-Jorge MH, Laurenti R, Gotlieb SLD. Análise da qualidade das estatísticas vitais brasileiras: a experiência de implantação do SIM e do SINASC. Ciênc Saúde Coletiva 2007; 12:643-54.

3. Andrade CLT, Szwarcwald CL. Desigualdades sócio-espaciais da adequação das informações de nascimentos e óbitos do Ministério da Saúde, Brasil, 2000-2002. Cad Saúde Pública 2007; 23: 1207-16.

4. Lima CRA, Schramm JMA, Coeli CM, Silva MEM. Revisão das dimensões de qualidade dos dados e métodos aplicados na avaliação dos sistemas de informação em saúde. Cad Saúde Pública 2009; 25:2095-109.

5. Correa PRL, Assunção AA. A subnotificação de mortes por acidente de trabalho: estudo de três bancos. Epidemiol Serv Saúde 2003; 12:203-12.

6. Haraki CAC, Gotlieb SLD, Laurenti R. Confiabilidade do Sistema de Informações sobre Mortalidade em município do sul do Estado de São Paulo. Rev Bras Epidemiol 2005; 8:19-24.
7. Szwarcwald CL, Leal MC, Andrade CLT, Souza Jr. PRB. Estimação da mortalidade infantil no Brasil: o que dizem as informações sobre óbitos e nascimentos do Ministério da Saúde? Cad Saúde Pública 2002; 18:1725-36.

8. Campos D, França E, Loschi RH, Souza MFM. Uso da autópsia verbal na investigação de óbitos com causa mal definida em Minas Gerais, Brasil. Cad Saúde Pública 2010; 26:1221-33.

9. Frias PG, Pereira PMH, Andrade CLT, Szwarcwald CL. Sistema de Informações sobre Mortalidade: estudo de caso em municípios com precariedade dos dados. Cad Saúde Pública 2008; 24:2257-66.

10. Frias PG, Vidal SA, Pereira PMH, Lira PIC, Vanderlei LC. Avaliação da notificação de óbitos infantis ao Sistema de Informações sobre Mortalidade: um estudo de caso. Rev Bras Saúde Matern Infant 2005; 5 Suppl 1:S43-52.

11. Cunha CC, Campos D, França EB. Uso da busca ativa de óbitos na avaliação do Sistema de Informações sobre Mortalidade em Minas Gerais, Brasil. Epidemiol Serv Saúde 2011; 20:275-86. 
12. Szwarcwald CL, Morais Neto OL, Frias PG, Souza Jr. PRB, Cortez-Escalante JJ, Lima RB, et al. Busca ativa de óbitos e nascimentos no Nordeste e na Amazônia Legal: estimação das coberturas do SIM e do SINASC nos municípios brasileiros. In: Departamento de Análise de Situação de Saúde, Secretaria de Vigilância em Saúde, Ministério da Saúde, organizadores. Saúde Brasil, 2010: uma análise da situação de saúde e de evidências selecionadas de impacto de ações de vigilância em saúde. Brasília: Ministério da Saúde; 2011. p. 79-98. (Série G. Estatísticas e Informação em Saúde).

13. Almeida MF. Descentralização de sistemas de informação e o uso das informações a nível municipal. Inf Epidemiol SUS 1998; 7:27-33.

14. Ministério da Saúde. Regulamento: pactos pela vida e de gestão. v. 2. Brasília: Ministério da Saúde; 2006. (Série A. Normas e Manuais Técnicos).

15. Frias PG, Pereira PMH, Szwarcwald CL, Lira PIC. Estimação da mortalidade infantil no contexto de descentralização do Sistema Único de Saúde (SUS). Rev Bras Saúde Matern Infant 2011; 11: 463-70.

16. Simões CC. Estimativas da mortalidade infantil por microrregião e municípios. Brasília: Ministério da Saúde; 1999.

17. Secretaria de Vigilância em Saúde, Ministério da Saúde. Instrutivo para o preenchimento da Programação das Ações de Vigilância em Saúde nas unidades federadas - 2010-2011. http://portal. saude.gov.br/portal/arquivos/pdf/instrutivo_pre enchimento_pavs_2010_2011.pdf (acessado em 10/Jan/2011).

18. Frias PG, Pereira PMH, Andrade CLT, Lira PIC, Szwarcwald CL. Avaliação da adequação das informações de mortalidade e nascidos vivos no Estado de Pernambuco, Brasil. Cad Saúde Pública 2010; 26:671-81.

19. Paes NA. Avaliação da cobertura dos registros de óbitos dos Estados brasileiros em 2000. Rev Saúde Pública 2005; 39:882-90.

20. Mello-Jorge MHP, Laurenti R, Gotlieb SLD. O Sistema de Informações sobre Mortalidade - SIM: concepção, implantação e avaliação. In: Ministério da Saúde; Organização Pan-Americana da Saúde; Fundação Oswaldo Cruz, organizadores. A experiência brasileira em sistemas de informação em saúde: produção e disseminação sobre saúde no Brasil. v. 1. Brasília: Ministério da Saúde; 2009. p. 71-96. (Série B. Textos Básicos de Saúde).
21. Façanha MC, Pinheiro AC, Fauth S, Lima AWDBC, Silva VLP, Justino MWS, et al. Busca ativa de óbitos em cemitérios da região metropolitana de Fortaleza, 1999 a 2000. Epidemiol Serv Saúde 2003; 12:131-6.

22. Silveira MH, Laurenti R. Os eventos vitais: aspectos de seus registros e inter-relação da legislação vigente com as estatísticas de saúde. Rev Saúde Pública 1973; 7:37-50.

23. Rattner DA. Epidemiologia na avaliação da qualidade: uma proposta. Cad Saúde Pública 1996; 12 Suppl 2:21-32.

24. Fundação Nacional de Saúde, Ministério da Saúde. Manual de procedimentos do Sistema de Informação sobre Mortalidade. Brasília: Ministério da Saúde; 2001.

25. Secretaria de Vigilância em Saúde, Ministério da Saúde. Portaria no 20, de 3 de outubro de 2003. Regulamenta a coleta de dados, fluxo e periodicidade de envio das informações sobre óbitos e nascidos vivos para os sistemas de informações em saúde SIM e SINASC. Diário Oficial da União 2003; 9 out.

26. Secretaria de Vigilância em Saúde, Ministério da Saúde. Portaria no 116, de 11 de fevereiro de 2009. Regulamenta a coleta de dados, fluxo e periodicidade de envio das informações sobre óbitos e nascidos vivos para os sistemas de informações em saúde sob gestão da Secretaria de Vigilância em Saúde. Diário Oficial da União 2009; 12 fev.

27. Vanderlei LC, Arruda BKG, Frias PG, Arruda S. Avaliação da qualidade de preenchimento das declarações de óbito em unidades terciária de atenção à saúde materno-infantil. Inf Epidemiol SUS 2002; 11:7-14.

28. Coeli CM, Coutinho ESF, Veras RP. O desafio da aplicação da metodologia de captura-recaptura na vigilância do diabetes mellitus em idosos: lições de uma experiência no Brasil. Cad Saúde Pública 2004; 20:1709-20.

29. Schmid B, Silva NN. Estimação de sub-registro de nascidos vivos pelo método de captura e recaptura, Sergipe. Rev Saúde Pública 2011; 45:1088-98.

Recebido em 08/Mar/2012

Versão final reapresentada em 06/Nov/2012 Aprovado em 16/Nov/2012 